\title{
On the Integration of Autonomous Data Marts
}

\author{
Luca Cabibbo and Riccardo Torlone \\ Dipartimento di Informatica e Automazione \\ Università di Roma Tre \\ \{cabibbo,torlone\}@dia.uniroma3.it
}

\begin{abstract}
We address the problem of integrating a federation of dimensional data marts. This problem arises when, e.g., a large organization (or a federation thereof) needs to combine independently developed data warehouses. We show that this problem can be tackled in a systematic way because of two main reasons. First, data marts are structured in a rather uniform way, along dimensions and facts. Second, data quality in data marts is usually higher than in generic databases, since they are obtained by reconciling several data sources. Our scenario of reference is a federation (i.e., a logical integration) of various data marts, which we need to query in a unified way, that is, by means of drill-across operations. We propose a novel notion of dimension compatibility and characterize its general property. We then show the significance of dimension compatibility in performing drill-across queries over autonomous data marts. We also discuss general strategies for the integration of data marts.
\end{abstract}

\section{Introduction}

Data warehousing can be considered today a mature technology. Many advanced tools exists to support business analysts in the rapid construction, the effective maintenance and the efficient analysis of data marts, the building blocks of a data warehouse [7]. As it often happens however, the rapid and uncontrolled spreading of these tools within organizations has led in many cases a number of previously uncharted and rather involved challenges.

One problem that occurs in practice is the integration of autonomous (i.e. independently developed and operated) data marts. A data mart provides a dimensional view of a single business process and it is today a common practice to build a data warehouse as a series of integrated data marts. According to Kimball [7], a key success factor in building data marts that form a data warehouse is to use a bus architecture based on conformed (i.e., common) dimen- sions and facts. In large companies however, very often different departments develop their data marts independently, and it turns out that their integration is a difficult task. Actually, the need of combining autonomous data marts arises in other common cases, for instance, when companies merge or get involved in a federated project. Another common scenario requiring data mart integration occurs when we wish to combine a proprietary data warehouse with multidimensional data available on the Web [12].

Differently from the general problem of database integration [5], we believe that the integration of data marts can be tackled in a more systematic way because of two important reasons. First, any data mart is structured in a rather uniform way, along the widely accepted notions of dimension and fact. Second, data quality in a data mart is usually higher than in a generic database, since it is obtained by cleaning and reconciling several data sources. These observations suggest that the problem of integrating autonomous data marts can be mainly focused on the integration of independently developed facts and dimensions.

According to this view, in this paper we introduce and investigate a fundamental notion underlying data mart integration: dimension compatibility. Intuitively, two dimensions (belonging to different data marts) are compatible when their common information is consistent. Similarly, two facts are compatible when their contents can be combined in a meaningful way. Having compatible dimensions and facts is important because it gives the ability to look consistently at data across data marts and to combine and correlate such data, e.g., to perform value chain analyses. In particular, drill-across queries are based on joining multiple data marts over common dimensions [7]. For example, assume to have three data marts, describing promotions of products, sales of products, and store inventory levels of products, respectively. A drill-across query over all these three data marts is needed if we want to identify products that, although in promotion and available in the stores, have sold under expectations. For a drill across query, compatibility of dimensions and facts is required to obtain meaningful results. We show that drill across operations over in- 
compatible dimensions and facts are not possible or, worse, produce invalid results. To this end, we first introduce a dimension algebra that allows us to select the relevant portion of a dimension for integration purposes. We then discuss integration strategies based on the inspection and the enforcement of dimension compatibility.

This paper provides the foundations for our ultimate goal: the development of a system for supporting the complex tasks related to the integration of autonomous data marts, similarly to how Clio [10] supports heterogeneous data transformation and integration.

The integration of heterogenous databases has been extensively studied in the literature (surveys on the many facets of this issue can be found in $[5,6,8,11,14])$. In this paper we take apart the general aspects of the problem and concentrate our attention on multidimensional data integration. This subject has been studied by Kimball [7] in the context of data warehouse design. In his book, he has identified the problem and has introduced, in an informal way, the notions of dimension and fact conformity. Our notion of compatibility has been inspired by this work, but extends and formalizes Kimball's notion of conformity in a way that, we believe, is more suitable to autonomous data mart integration. Abellò et al. [1] have investigated four kinds of relationships among dimensions and facts (derivation, generalization, association, and flow) that are relevant to drillacross navigation. Although our notion of compatibility is related to these kinds of relationships, the goals of the papers differ, since we refer to drill-across queries whereas [1] refers a weaker form of drill acrossing, specific to interactive navigation. Some work has been done on the problem of integrating data marts with external data stored in various formats: object-oriented [13] and XML [12]. This is clearly related to but different from our goal, since no attempt is made to combine multiple multidimensional databases.

The rest of the paper is organized as follows. In Section 2 we recall $\mathcal{M D}$, a conceptual model for multidimensional data, introduced in [3], that will be used throughout this paper. In Section 3 we present an algebra over dimensions, a tool that will be used, in Section 4, to introduce the notion of dimension compatibility. In Section 5 we investigate the relationship between compatibility and the operation of drill across between data marts. In Section 6 we discuss possible strategies for autonomous data mart integration and finally, in Section 7, we sketch some conclusions and discuss future work directions of research.

\section{A dimensional data model}

In this section, we describe the $\mathcal{M D}$ data model [3], a multidimensional conceptual data model that will be used throughout this paper. This choice is motivated by the fact that $\mathcal{M D}$ includes a number of concepts that generalize the notions commonly used in multidimensional analysis or available in commercial OLAP systems, e.g., dimensions, fact tables [7] and cubes [9]. Because of this, our approach can be considered rather general and can be applied to other multidimensional data models [15].

$\mathcal{M D}$ is based on two main constructs: the dimension and the f-table. A dimension represents a domain of real-world entities called members. Members of a dimension can be the days in a time interval, the products sold by a company, or a collection of stores selling these products. Each dimension is organized into a hierarchy of levels, corresponding to data domains grouping dimension members at different granularity. For example, products can be grouped into brands and categories, and days can be grouped into months and years. Within a dimension, members at different levels are related through a family of roll-up functions. A roll-up function relates the members of a pair of levels by mapping each member having a finer grain (e.g., a product) to a member having a coarser grain (e.g., a brand). An f-table is the conceptual counterpart of a fact table and associate measures to members of dimensions and are used to represent factual data. For example, the daily sales of a chain of stores can be represented by an f-table that associates with a product, a day, and a store, the number of items sold of that product, day, and store, together with the corresponding gross income and cost. In the following, we provide a more systematic presentation of these notions.

Definition 2.1 (Dimension) A dimension $d$ is composed of:

- a scheme, made of:

- a finite set $L(d)=\left\{l_{1}, \ldots, l_{n}\right\}$ of levels; and

- a partial order $\preceq_{d}$ on $L(d)$; if $l_{1} \preceq_{d} l_{2}$ we say that $l_{1}$ rolls up to $l_{2}$;

- an instance, made of:

- a function $m$ associating a set of members with each level; and

- a family of functions $\rho$ including a roll up function $\rho^{l_{1} \rightarrow l_{2}}: m\left(l_{1}\right) \rightarrow m\left(l_{2}\right)$ for each pair of levels $l_{1} \preceq l_{2}$.

We assume that $L(d)$ contains a bottom element $\perp_{d}$ (wrt $\preceq_{d}$ ). We shall simply write $\preceq$ instead of $\preceq_{d}$ whenever $d$ is clear from the context.

Actually, each member of the bottom level $\perp_{d}$ of a dimension $d$ (the finest grain for the dimension) represents a real world entity that we call ground. Members of other levels represent groups of ground members. For example, within a dimension of products, a ground member is a single product, whereas a member of the level brand describes a single brand, that is, the group composed by all the products of that brand. The active domain of a dimension $d$ is 
the set of all the members that actually belong to the various levels of $d$.

For each pair of levels $l_{1}, l_{2}$ of a dimension $d$ such that $l_{1} \preceq_{d} l_{2}$, we assume that the following holds in any instance:

- if $o_{1} \in m_{d}\left(l_{1}\right)$, then there is an $o_{2} \in m_{d}\left(l_{2}\right)$ such that $\rho^{l_{1} \rightarrow l_{2}}\left(o_{1}\right)=o_{2}$;

- if $o_{2} \in m_{d}\left(l_{2}\right)$, then there exists at least an $o_{1} \in$ $m_{d}\left(l_{1}\right)$ such that $\rho^{l_{1} \rightarrow l_{2}}\left(o_{1}\right)=o_{2}$.

Therefore, for each ground member of the dimension there is a member in each aggregation level to which it rolls up. Furthermore, for each member of a non-bottom level, there is at least a ground member that rolls up to it.

Let $\left\{\tau_{1}, \ldots, \tau_{k}\right\}$ be a predefined set of base types, (including integers, real numbers, etc.).

Definition 2.2 (F-table) An $f$-table $f$ over a set $D$ of dimensions is composed of:

- a scheme $f\left[A_{1}: l_{1}, \ldots, A_{n}: l_{n}\right] \rightarrow\left\langle M_{1}:\right.$ $\left.\tau_{1}, \ldots, M_{m}: \tau_{m}\right\rangle$, where each $A_{i}$ is a distinct attribute name, each $l_{i}$ is a level of some dimension in $D$, each $M_{j}$ is a distinct measure name, and each $\tau_{j}$ is some base type; and

- an instance, which is a partial function mapping coordinates for $f$ to facts for $f$, where:

- a coordinate is a tuple over the attributes of $f$, that is, a function mapping each attribute name $A_{i}$ to a member of $l_{i}$;

- a fact is a tuple over the measures of $f$, that is, a function mapping each measure name $M_{j}$ to a value in the domain of type $\tau_{j}$.

A collection of f-tables over the same dimensions compose a data mart.

Definition 2.3 (Data mart) A data mart is composed of:

- a set $D$ of dimensions; and

- a set $F$ of f-tables over the dimensions in $D$.

Example 2.4 Figures 1, 2, and 3 show three (autonomous) data marts, each consisting of a single f-table. This example is inspired by case studies discussed in [7]. The Sales data mart (Figure 1) represents daily sales of products in a chain of stores. The Store Inventory data mart (Figure 2) represents inventory snapshots for the same products and stores of the Sales data mart given on a weekly base. Finally, the Warehouse Inventory data mart (Figure 3) represents daily inventory snapshots for the warehouses supplying only the food and beverage products to the same stores of the Sales and Store Inventory data marts.
Note that the granularity of data in the three data marts is different. This is apparent for time data, given daily in two data marts, but weekly in the other one. Similarly, products are described as SKUs (stock keeping units, that is, products that can be sold at retail, like a can of coke) at the stores, but as packages (boxes of SKUs, e.g., a package of 20 cans of coke) at the warehouses.

Another difference is that the first two data marts contain data about many products along years 2000-2003, whereas the warehouse data mart contains only data about food and beverage products along years 2002-2003.

It is worth noting that, according to the traditional database terminology, the $\mathcal{M D}$ is a conceptual data model and therefore its schemes can be implemented using several logical data model [2]. For example, as described in [3], an $\mathcal{M D}$ data mart can be easily implemented as a set of star schemes [7] having a dimension table for each dimension and a fact table for each f-table. Each ground member of a dimension would be represented by a tuple in the corresponding dimension table. Each symbolic coordinate and the associated fact of an f-table would be represented by a tuple in the corresponding fact table.

\section{An algebra for dimensions}

We now introduce the dimension algebra (DA), a simple algebra over dimensions that will be used to extract subdimensions from a given dimensions. Specifically, DA is based on three operators: (i) selection, which restricts a dimension to a subset of its ground members; (ii) projection, which prunes levels and roll-up functions from a dimension; (iii) aggregation, which aggregates over a level in a dimension.

In what follows, $d$ denotes a dimension having scheme $(L(d), \preceq)$ and instance $(m, \rho)$.

Definition 3.1 (Selection) Let $S$ be a subset of the ground members of $d$. The selection $\sigma_{S}(d)$ of $d$ over $S$ is the dimension $d^{\prime}$ such that: (i) the scheme of $d^{\prime}$ is the same of $d$ and (ii) the instance of $d^{\prime}$ contains: the ground members in $S$, the members of $d$ that can be reached from them by applying roll-up functions in $\rho$, all the roll-up functions of $d$ restricted to the members of $d^{\prime}$.

Definition 3.2 (Projection) Let $X$ be a subset of the scheme of $d$ such that: (i) $\perp_{d} \in X$ and (ii) if $X$ contains $l_{1} \preceq l_{2}$ then both $l_{1}$ and $l_{2}$ are in $X$. The projection $\pi_{X}(d)$ of $d$ over $X$ is the dimension $d^{\prime}$ such that: (i) the scheme of $d^{\prime}$ is $X$ and (ii) the instance of $d^{\prime}$ contains: only the members of $d$ that belong to levels in $X$ and only the roll-up functions $\rho^{l_{1} \rightarrow l_{2}}$ of $d$ such that $l_{1} \preceq l_{2}$ belong to $X$. 


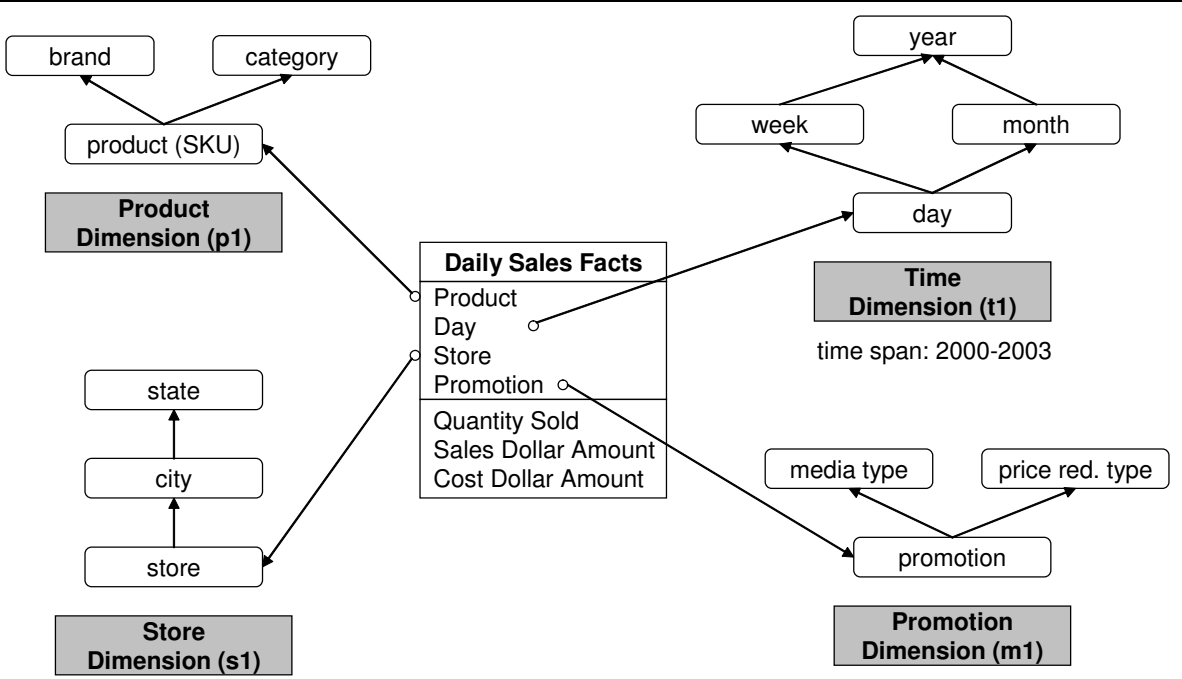

Figure 1. Sales data mart

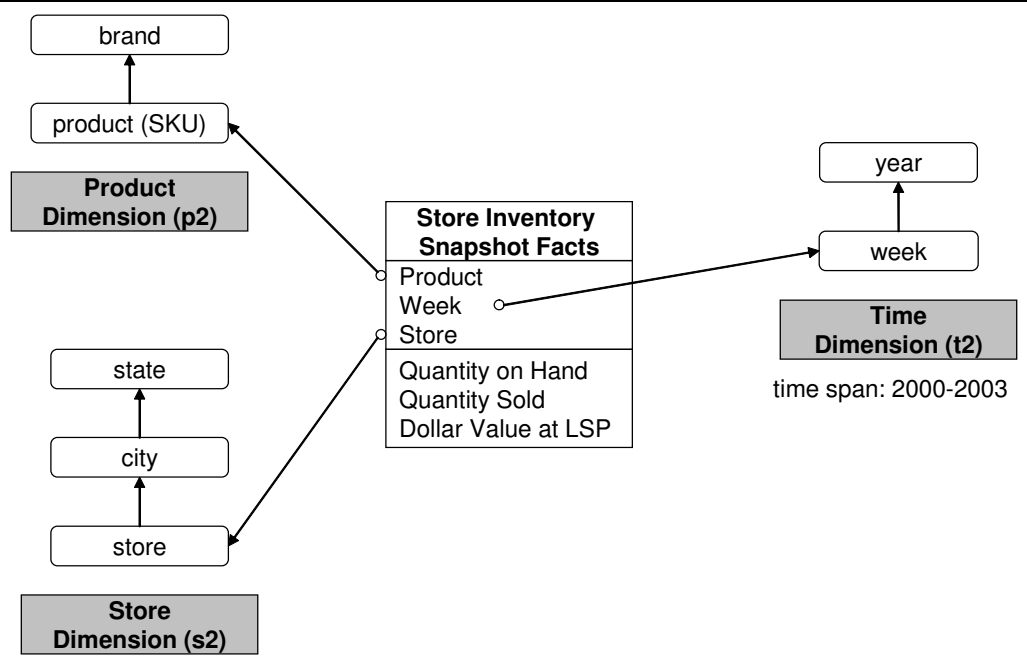

Figure 2. Store Inventory data mart

Definition 3.3 (Aggregation) Let $l$ be a level in $L(d)$. The aggregation $\psi_{l}(d)$ of $d$ over $l$ is the dimension $d^{\prime}$ such that: (i) the scheme of $d^{\prime}$ contains $l$, all the levels of $d$ to which $l$ rolls up, and the restriction of $\preceq$ to these levels, and (ii) the instance of $d^{\prime}$ contains: only the members of $d$ that belong to levels in $d^{\prime}$ and only the roll-up functions $\rho^{l_{1} \rightarrow l_{2}}$ of $d$ such that $l_{1} \preceq l_{2}$ belong to $d^{\prime}$.

The simplest DA expression consists just of a dimension name, and its result is the dimension itself. More complex DA expressions can be written by applying and combining the three DA operators above to a dimension. For a DA expression $E$ and a dimension $d$, we denote by $E(d)$ the dimension obtained by applying $E$ to $d$.
Example 3.4 In Example 2.4, the Time dimension $t_{2}$ of the Store Inventory data mart can be computed by applying an aggregation to the Time dimension $t_{1}$ of the Sales data mart, as follows (see Figure 4):

$$
\psi_{\text {week }}(d) .
$$

Similarly, the Time dimension $t_{3}$ of the Warehouse Inventory data mart can be computed by applying a selection followed by a projection to the Time dimension $t_{1}$ of the Sales data mart, as follows (see Figure 5):

$$
\pi_{\text {day,month,year,day } \preceq \text { month,month } \preceq \text { year }}\left(\sigma_{O_{2002-2003}}(d)\right) \text {, }
$$

where $O_{2002-2003}$ denotes the days that belong to years 2002-2003. 


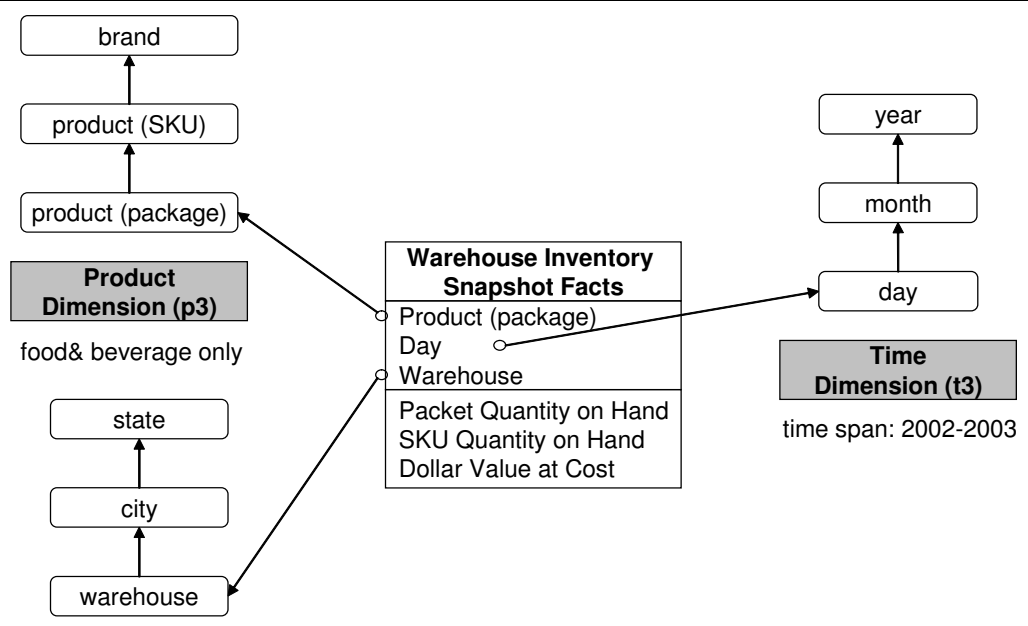

Warehouse

Dimension (w3)

Figure 3. Warehouse Inventory data mart
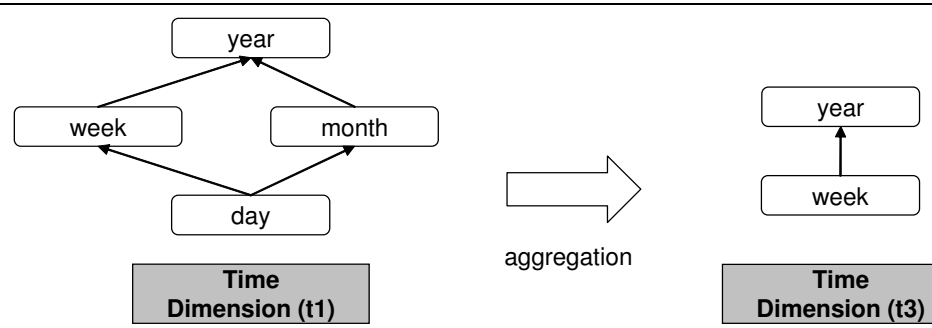

aggregation

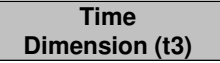

time span: $2000-2003$

time span: $2000-2003$

Figure 4. Application of a DA expression

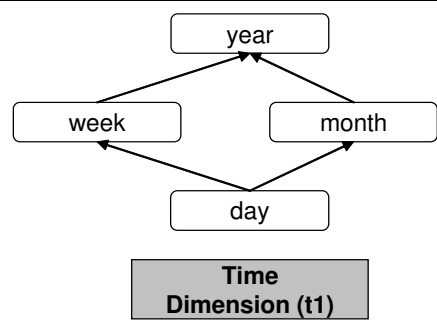

time span: $2000-2003$

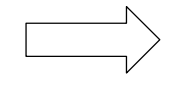

selection and projection

time span: 2002-2003

Figure 5. Application of another DA expression 
It is worth noting that DA expressions allow to "reduce" the scheme and/or the instance of a dimension. Indeed, the goal of a DA expression is to compute a subset of a dimension. Note also that projection and aggregation have different goals, since projections always keep the bottom level whereas non trivial aggregations drop it.

We now introduce a desirable property of DA expressions.

Definition 3.5 (Lossless expression) A DA expression $E$ over a dimension $d$ is lossless if, for each each pair of ground members $o_{1}, o_{2} \in m_{d}\left(\perp_{d}\right)$ and for each level $l \in L(d)$ such that $o_{1}$ and $o_{2}$ roll up to a same member $o \in m_{d}(l)$ (that is, $\rho_{d}^{\perp_{d} \rightarrow l}\left(o_{1}\right)=\rho_{d}^{\perp_{d} \rightarrow l}\left(o_{2}\right)=o$ ), then neither or both $o_{1}, o_{2}$ belong to the active domain of $E(d)$.

In other words, $E$ is lossless if, whenever a member $o$ belongs to $E(d)$, then all the members that roll up to $o$ in $d$ belong to $E(d)$ as well. This property is important because, if $E$ is lossless, then aggregating an f-table over $E(d)$ yields as result a subset of the facts that can be obtained by aggregating over $d$, with the same measures. Otherwise, the result of aggregating over $E(d)$ could produce different results than aggregating directly over $d$.

Proposition 3.6 DA expressions involving only projections and aggregations are always lossless.

On the other hand, if a DA expression involves selections, the lossless property can fail to hold: it depends on the particular sets of elements chosen to perform the selections.

\section{Dimension compatibility}

In this section we present our notion of compatibility among dimensions. Intuitively, two dimensions are compatible if their share some information and this common information is consistent. This is a very important requirement in drill across queries, where data marts are joined over related dimensions.

The notion of compatibility between dimensions will be introduced gradually, by first defining the stronger notion of equivalence. In what follows, $d_{1}$ and $d_{2}$ denote two dimensions, belonging to different data marts, having scheme $\left(L\left(d_{i}\right), \preceq d_{i}\right)$ and instance $\left(m_{i}, \rho_{i}\right)$, respectively. Moreover, $l_{1}$ and $l_{2}$ denote two levels, $l_{1} \in L\left(d_{1}\right)$ and $l_{2} \in L\left(d_{2}\right)$.

Definition 4.1 (Level equivalence) Two levels $l_{1}$ and $l_{2}$ are equivalent (written $l_{1} \equiv l_{2}$ ) if they have the same members, that is, $m_{1}\left(l_{1}\right)=m_{2}\left(l_{2}\right)$.

Definition 4.2 (Dimension equivalence) Two dimensions $d_{1}$ and $d_{2}$ are equivalent (written $d_{1} \equiv d_{2}$ ) if there exists a bijection $\phi$ between $L\left(d_{1}\right)$ and $L\left(d_{2}\right)$ such that:
- for each level $l \in L\left(d_{1}\right), l$ is equivalent to $\phi(l)$;

- for each pair of levels $l, l^{\prime} \in L\left(d_{1}\right), l \preceq d_{1} l^{\prime}$ if and only if $\phi(l) \preceq d_{2} \phi\left(l^{\prime}\right)$; and

- for each pair of levels $l, l^{\prime} \in L\left(d_{1}\right)$ such that $l \preceq d_{1} l^{\prime}$, the roll-up functions $\rho_{1}^{l \rightarrow l^{\prime}}$ and $\rho_{2}^{\phi(l) \rightarrow \phi\left(l^{\prime}\right)}$ are equal.

According to this definition, two equivalent dimensions represent exactly the same information, apart from differences in the choice of the names given to levels. It is worth noting that our notion of equivalence is conceptual. Under this view, two levels are equivalent if they are populated by the same real world entities. In a logical approach, in which entities are represented by their identifiers, level equivalence would be based on a bijection between such identifiers.

It is still possible that two non-equivalent dimensions have some information in common. The first requirement is the existence of an operational way to compare portions of dimensions. This comment leads to the following definitions.

Definition 4.3 (Dimension comparability) Two dimensions $d_{1}$ and $d_{2}$ are comparable if there exist DA expressions $E_{1}$ and $E_{2}$ over $d_{1}$ and $d_{2}$, respectively, such that $E_{1}\left(d_{1}\right)$ and $E_{2}\left(d_{2}\right)$ are not empty and equivalent. In this case, we say that $d_{1}$ and $d_{2}$ are comparable using $E_{1}$ and $E_{2}$.

Definition 4.4 (Dimension intersection) If two dimensions $d_{1}$ and $d_{2}$ are comparable using $E_{1}$ and $E_{2}$, then the dimension $E_{1}\left(d_{1}\right) \equiv E_{2}\left(d_{2}\right)$ is called an intersection of $d_{1}$ and $d_{2}$.

We are now ready to introduce our notion of compatibility between dimensions.

Definition 4.5 (Dimension compatibility) Two dimensions $d_{1}$ and $d_{2}$ are compatible if they are comparable using two lossless DA expressions $E_{1}$ and $E_{2}$.

In sum, the rationale under the definition of compatibility is that: (i) the intersection of two dimensions represents their common information; (ii) DA expressions are used to compute this intersection; and (iii) lossless expressions avoid inconsistency, in a sense that will be clarified in the following section.

Example 4.6 Consider again the data marts of Example 2.4. The Store dimensions $s_{1}$ and $s_{2}$ of the Sales and Store Inventory data marts are equivalent. On the other hand, their Product dimensions $p_{1}$ and $p_{2}$ are not equivalent but compatible; in fact, $p_{2}$ can be computed from $p_{1}$ as $\pi_{\text {product }(S K U), \text { brand,product }(S K U) \preceq \text { brand }}\left(p_{1}\right)$. The Time dimensions $t_{1}$ and $t_{2}$ are also compatible: $t_{2}$ can be computed 
as $\psi_{\text {week }}\left(t_{1}\right)$. The Store dimension $s_{1}$ and the Warehouse dimension $w_{3}$ are compatible, since $\psi_{\text {city }}\left(s_{1}\right)$ is equivalent to $\psi_{\text {city }}\left(w_{3}\right)$. The Product dimensions $p_{1}$ and $p_{3}$ are compatible too: their common part can be computed by aggregating $p_{3}$ over level product $(S K U)$, and by applying to $p_{1}$ a projection (over levels product (SKU) and brand and the roll-up relationship between them) and a selection (over food and beverage products).

\section{Drill across queries and dimension compat- ibility}

In this section we investigate the impact of dimension compatibility on drill across queries.

We first define a drill across operator. In [4] we have defined a natural join operation $f_{1} \bowtie f_{2}$ of two f-tables over a set of common attributes (defined on the same dimensions) whose result is the f-table having as entries the natural join (in the relational sense) of the entries of $f_{1}$ and $f_{2}$ and as facts (i.e., measures) the juxtaposition of their facts. A drill across operation between two f-tables $f_{1}$ and $f_{2}$ can be defined as an extension of the natural join, in which common attributes refer to different but compatible dimensions. In this case, before joining $f_{1}$ and $f_{2}$, for each pair of common attributes over compatible dimensions $d_{1}$ and $d_{2}$, we identify an intersection $d_{1 \cap 2}$ of $d_{1}$ and $d_{2}$ and then aggregate $f_{1}$ and $f_{2}$ over the bottom level of $d_{1 \cap 2}$.

Example 5.1 Consider again the data marts in Example 2.4 and a drill across operation over the Sales and Store Inventory data marts. They can be combined over the compatible dimensions Product, Time, and Store. It follows that the drill across requires an aggregation of the Sales data mart over the Time dimension at the week level.

Another possible drill across query is on the Sales and Warehouse Inventory data marts. Before joining them, they need to be aggregated over the common city level in the compatible dimensions Store and Warehouse.

A number of anomalies can arise in the computation of a drill across query:

- some detail of the original data marts can be lost in the aggregations preceding the join, when f-tables store facts at different levels of aggregation;

- some data of the original data marts can be lost in the join, when f-tables refer to members that do not belong to the intersection of compatible dimensions.

As an example of the former type of anomaly, the drill across query over the Sales and Store Inventory data marts of Example 5.1 could retrieve weekly but not daily data. As an example of the latter kind of anomaly, the drill across query over the Sales and Warehouse Inventory data marts of Example 5.1 retrieves only data about food and beverage products along years 2002-2003 (see Example 2.4).

Both cases however refer to a loss of information that is not present in one of the original data marts. Therefore, these anomalies can be tolerated since they correspond to the generation of incomplete but correct results.

To understand the impact of dimension compatibility in drill across queries, let us consider a drill across query involving two comparable but incompatible dimensions $d_{1}$ and $d_{2}$. According to our definitions, this means that we are able to find data in common between $d_{1}$ and $d_{2}$, but the operations required to select these data produce some loss in the original dimensions that prevent the correctness of the result of the drill across query. More precisely, incompatibility implies that an aggregation over the original data marts can differ from the computation of the same aggregation over the result of the drill across query.

Example 5.2 Consider two data marts, one representing the costs of buying a set of products and the second the incomes in selling another set of products. Assume also that the two sets of products are different but overlapping. If we drill across over the two data marts, data is meaningful only for the common products. If we aggregate this data at, e.g., the category level, the costs and incomes obtained for each category are different from those that can be computed over the two individual data marts.

It is clear that this situation cannot be accepted in drill across queries over autonomous data marts. Dimension compatibility allows us to prevent this anomaly.

We now briefly discuss a problem related to measure (fact) compatibility. Intuitively, two measures of different data marts are compatible when their values can be combined (e.g., compared, added, or multiplied) in some meaningful way. This is very important in drill across queries. However, the characterization of measure compatibility requires a deep understanding of the semantics of measures and aggregate functions, as shown by the following example.

Example 5.3 Consider the second drill across query proposed in Example 5.1, over the Sales and Store Inventory data marts. Their dimensions Store and Warehouse are compatible at the city level. However, joining the two data marts on this common level is meaningful only if a business rule states that each warehouse in a city supply all and only stores in the same city. If this is not the case, the drill across over the city level is not correct, since the result of the query will contain meaningless facts. One possible solution could be to exclude the dimensions Store and Warehouse from the drill across query. 
It is worth noting that dimension compatibility is an extension of Kimball's dimension conformity [7], since dimension conformity implies dimension compatibility, but the converse does not hold in general. We also believe that, in autonomous data mart integration, dimension compatibility can be achieved more often than dimension conformity, and therefore it should be considered the most common criteria when integrating autonomous data marts.

\section{Integration strategies}

We now briefly illustrate a methodology for integrating autonomous data marts that refers to the notion of conformity.

1. Data marts to be integrated are analyzed, to identify if and how their dimensions are compatible.

2. Semantic matching of compatible dimensions is checked, to verify whether their join is meaningful.

3. Incompatible but related dimensions are identified and, if possible, made compatible on the basis of external information.

Step 1 could be performed using an interactive, semiautomated tool for data mart integration, similar in spirit to Clio [10] and supporting a wide range of scheme-matching and data-mapping techniques. Step 2 has the goal of preventing the combination of compatible but semantically heterogenous dimensions, as discussed in Example 5.3. Step 3 is oriented towards the identification and enforcement of further matchings and compatibilities, based on inter-scheme knowledge. This is discussed in the following examples.

Example 6.1 Consider again the data marts of Example 2.4, and that the Store Inventory and Warehouse Inventory data marts can be joined at levels product (SKU), city, and year. Actually, it should be possible to join their Time dimensions at the week level rather than at the year level. In fact, it suffices to extend the Time dimension $t_{3}$ with a week level by associating each day with the corresponding week in the year.

The above example suggests that external information, if available, should be used in data mart integration, e.g., by adding descriptive data to members. This idea is applied again in the following and more involved example.

Example 6.2 Consider again the problem of integrating the Store Inventory and Warehouse Inventory data marts of Example 2.4 and assume that each store is supplied only by a single warehouse. In this case, this knowledge can be used to add a warehouse level to the Store dimension, and to make the dimensions Store and Warehouse compatible at the warehouse level.

Note, however, that because of autonomy, such extra knowledge should not be embedded in the original data marts, but it should be rather stored in a ad-hoc repository, managed by a sort of Federated Data Warehouse System.

\section{Conclusion}

In this paper we have investigated the problem of integrating autonomous data marts, with the goal of querying them using drill-across operations. To this aim, we have proposed a novel notion of dimension compatibility and related it to drill-across queries. It turns out the dimension compatibility is a necessary condition to obtain meaningful results. We have also discussed some strategies for the integration of data marts.

This paper provides the foundations for our ultimate goal: the development of a system for supporting the complex tasks related to the integration of autonomous data marts, similarly to how Clio [10] supports heterogeneous data transformation and integration. This can be based on semi-automatic, interactive tools for describing multiple data marts, for determining and verifying correspondences among their schemes and data, as well as for defining and verifying mappings among them. For instance, borrowing some ideas from $[12,13]$, "integration links" can be used to relate data in different data marts.

Several concepts related to dimension and fact compatibility have been introduced in this paper in a rather informal way. In the future, we plan to study these notions and their relationships with the problem of data mart integration in more depth, both from a theoretical and practical perspective.

\section{References}

[1] A. Abelló, J. Samos, and F. Saltor. On relationships Offering New Drill-across Possibilities. In ACM Fifth Int. Workshop on Data Warehousing and OLAP (DOLAP 2002), pages 7-13, 2002.

[2] P. Atzeni, S. Ceri, S. Paraboschi, and R. Torlone. Database Systems: concepts, languages and architectures. McGrawHill, 1999.

[3] L. Cabibbo and R. Torlone. A logical approach to multidimensional databases. In Sixth Int. Conference on Extending Database Technology (EDBT'98), Springer-Verlag, pages 183-197, 1998.

[4] L. Cabibbo and R. Torlone. From a procedural to a visual query language for OLAP. In Int. Conference on Scientific and Statistical Database Management (SSDBM'98), pages 74-83, 1998. 
[5] A. Elmagarmid, M. Rusinkiewicz, and A. Sheth. Management of Heterogeneous and Autonomous Database Systems. Morgan Kaufmann, 1999.

[6] R. Hull. Managing Semantic Heterogeneity in Databases: A Theoretical Perspective. In 16th ACM SIGACT SIGMOD SIGART Symp. on Principles of Database Systems, pages 5161, 1997.

[7] R. Kimball and M. Ross. The Data Warehouse Toolkit: The Complete Guide to Dimensional Modeling. John Wiley \& Sons, Second edition, 2002.

[8] M. Lenzerini. Data Integration: A Theoretical Perspective. In 21 st ACM SIGACT SIGMOD SIGART Symp. on Principles of Database Systems, pages 233-246, 2002.

[9] Microsoft OLE DB programmer's reference and data access SKD. Microsoft Corporation, 1998.

[10] R.J. Miller, M.A. Hernández, L.M. Haas, L. Yan, C.T.H. Ho, R. Fagin, and L. Popa. The Clio Project: Managing Heterogeneity. SIGMOD Record, 30(1): 78-83, 2001.

[11] R.J. Miller (editor). Special Issue on Integration Management. IEEE Bulletin of the Technical Committee on Data Engineering, 25(3), 2002.

[12] D. Pedersen, K. Riis, and T.B. Pedersen. XML-Extended OLAP Querying. In Int. Conference on Scientific and Statistical Database Management (SSDBM'02), pages 195-206, 2002.

[13] T.B. Pedersen, A. Shoshani, J. Gu, and C.S. Jensen. Extending OLAP Querying to External Object Databases. In Int. Conference on Information and Knowledge Management, pages 405-413, 2000.

[14] E. Rahm and P.A. Bernstein. A survey of approaches to automatic schema matching. VLDB Journal, 10(4):334-350, 2001.

[15] P. Vassiliadis and T.K. Sellis. A Survey of Logical Models for OLAP Databases. SIGMOD Record, 28(4):64-69, 1999. 